\title{
Teaching NeuroImages: An uncommon cause of carotid artery dissection
}

\author{
Fabry disease
}

Aikaterini Theodorou, MD, Lina Palaiodimou, MD, Panagiotis Kokotis, MD, PhD,

Marianna Papadopoulou, MD, PhD, Spyridon Fradelos, MD, MSc, FEBO, Adamantia Voudouri, MD, PhD, Christina Zompola, MD, Georgios Magoufis, MD, Chrysa Arvaniti, MD, PhD, Anastasios Bonakis, MD, PhD, and Georgios Tsivgoulis, MD, PhD, MSc, FESO

Neurology ${ }^{\circledR}$ 2020;95:e2711-e2713. doi:10.1212/WNL.0000000000010650

Figure 1 Digital subtraction angiography (DSA) findings
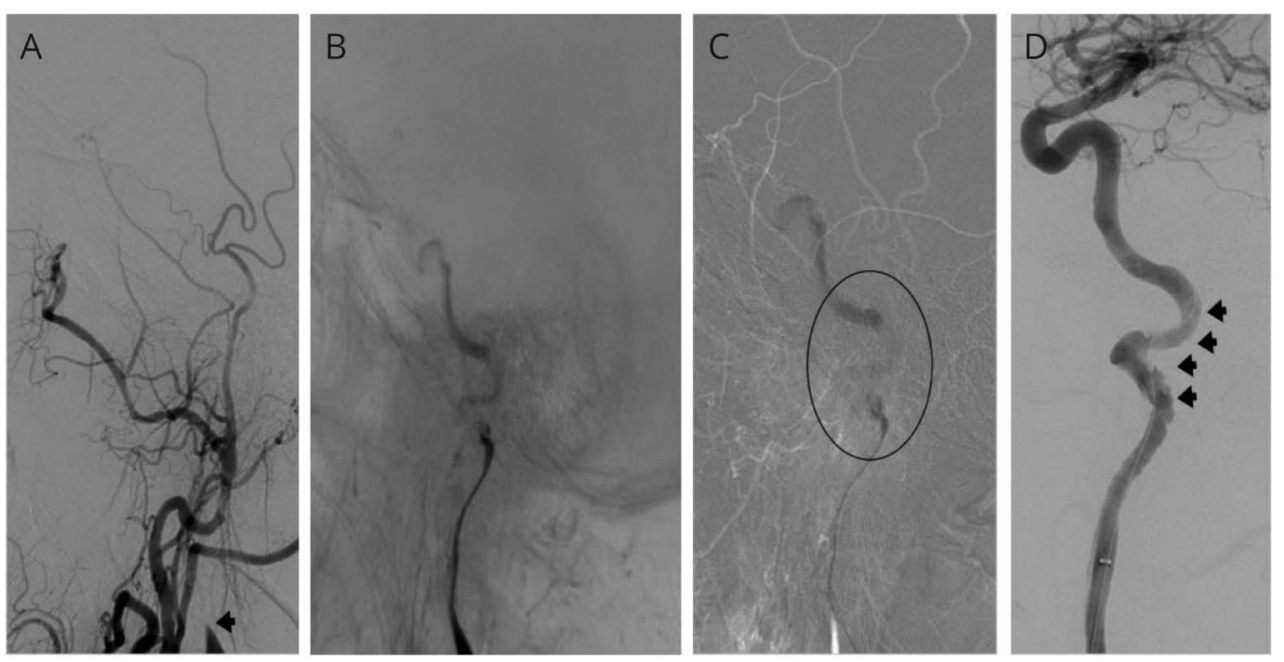

Diagnostic DSA reveals left cervical internal carotid artery occlusion due to underlying dissection with characteristic flame-shaped appearance (A). DSA images during $(B, C)$ and at the end of mechanical thrombectomy (D) show extracranial and intracranial internal carotid artery recanalization and mural dissection (D; arrows).

Cervical internal carotid artery dissection (CAD), a leading cause of ischemic stroke in young adults, has been rarely associated with Fabry disease (FD) ${ }^{1,2}$ A 49 -year-old man with left acute middle cerebral artery occlusion due to underlying spontaneous $\mathrm{CAD}$ received IV thrombolysis and mechanical thrombectomy, achieving complete reperfusion (figure 1).

Further diagnostic workup excluding other stroke etiologies revealed bilateral cornea verticillata and large-fiber sensory polyneuropathy with impaired lower limb temperature perception (figure 2, D-F). Low $\alpha$-galactosidase levels and molecular genetic testing disclosing pathogenic GLA variant (c427G >A p.[Ala143Thr]) established FD diagnosis.

This case highlights that FD may represent a rare cause of CAD due to sphingolipid accumulation in vessel walls.

\section{Correspondence}

Dr. Theodorou

katetheo24@gmail.com

\section{MORE ONLINE}

$\rightarrow$ Teaching slides

links.lww.com/WNL/

B191

From the Second Department of Neurology (A.T., L.P., M.P., C.Z., C.A., A.B., G.T.), National and Kapodistrian University of Athens, School of Medicine, "Attikon" University Hospital; Laboratory of Clinical Neurophysiology (P.K.), First Department of Neurology, School of Medicine, National and Kapodistrian University of Athens, "Aiginiteion" University Hospital; Department of Physiotherapy (M.P.), University of West Attica; Ophthalmological Center "Voudouri Adamantia" (S.F., A.V.), Athens; and Department of Interventional Neuroradiology (G.M.), Metropolitan Hospital, Piraeus, Greece.

Go to Neurology.org/N for full disclosures. Funding information and disclosures deemed relevant by the authors, if any, are provided at the end of the article. 
Figure 2 Diagnostic workup in Fabry disease
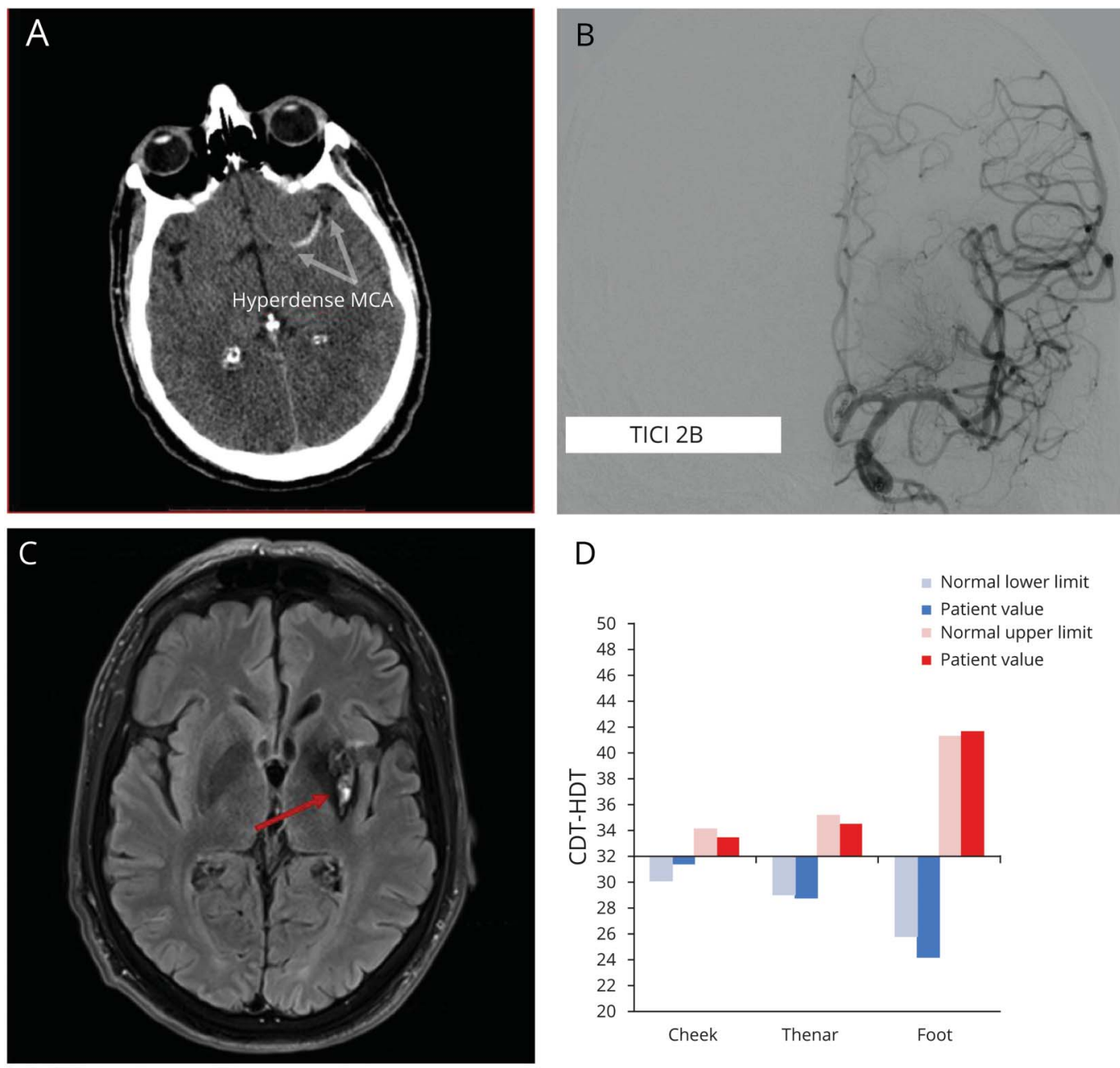

D

$\mathrm{E}$

- Normal lower limit

- Patient value

- Normal upper limit

- Patient value
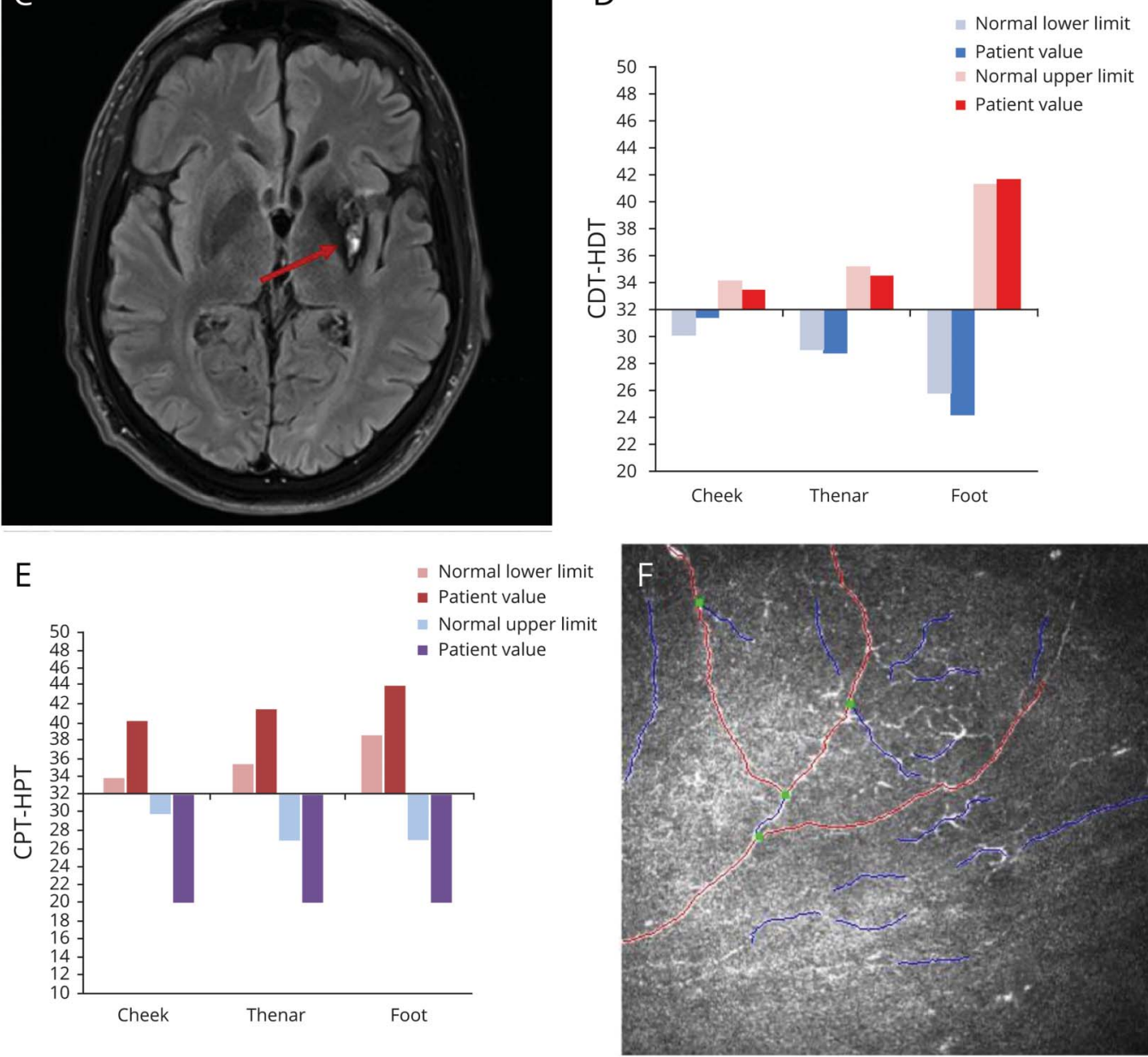

(A; arrows) Hyperdense middle cerebral artery (MCA) sign in the left M1 segment on baseline brain CT. (B) Post-thrombectomy digital subtraction angiography reveals complete reperfusion (Thrombolysis in Cerebral Infarction [TICI] 2B). (C; arrow) Residual basal ganglia infarction is depicted on follow-up brain MRI. (D, E) Quantitative sensory tests show impairment of cold and warm perception in the lower limbs and (F) corneal confocal microscopy reveals bilateral cornea verticillata. CDT-HDT = cold and heat detection thresholds. 


\section{Study funding}

No targeted funding reported.

\section{Disclosure}

The authors report no disclosures relevant to the manuscript. Go to Neurology.org/N for full disclosures.

\begin{tabular}{|c|c|c|}
\hline Name & Location & Contribution \\
\hline $\begin{array}{l}\text { Aikaterini } \\
\text { Theodorou, MD }\end{array}$ & $\begin{array}{l}\text { "Attikon" University } \\
\text { Hospital Athens, Greece }\end{array}$ & $\begin{array}{l}\text { Drafting and revising } \\
\text { the manuscript }\end{array}$ \\
\hline $\begin{array}{l}\text { Lina } \\
\text { Palaiodimou, } \\
\text { MD }\end{array}$ & $\begin{array}{l}\text { "Attikon" University } \\
\text { Hospital Athens, Greece }\end{array}$ & $\begin{array}{l}\text { Data collection, critical } \\
\text { comments during } \\
\text { manuscript revision }\end{array}$ \\
\hline $\begin{array}{l}\text { Panagiotis } \\
\text { Kokotis, MD, } \\
\text { PhD }\end{array}$ & $\begin{array}{l}\text { "Aiginiteion" University } \\
\text { Hospital, Athens, Greece }\end{array}$ & $\begin{array}{l}\text { Data collection, } \\
\text { critical comments } \\
\text { during manuscript } \\
\text { revision }\end{array}$ \\
\hline $\begin{array}{l}\text { Marianna } \\
\text { Papadopoulou, } \\
\text { MD, PhD }\end{array}$ & $\begin{array}{l}\text { University of West Attica, } \\
\text { Athens, Greece }\end{array}$ & $\begin{array}{l}\text { Critical comments } \\
\text { during manuscript } \\
\text { revision }\end{array}$ \\
\hline $\begin{array}{l}\text { Spyridon } \\
\text { Fradelos, MD, } \\
\text { MSc, FEBO }\end{array}$ & $\begin{array}{l}\text { Ophthalmologic Center } \\
\text { "Voudouri Adamantia," } \\
\text { Athens, Greece }\end{array}$ & $\begin{array}{l}\text { Data collection, critical } \\
\text { comments during } \\
\text { manuscript revision }\end{array}$ \\
\hline
\end{tabular}

Appendix (continued)

\begin{tabular}{|c|c|c|}
\hline Name & Location & Contribution \\
\hline $\begin{array}{l}\text { Adamantia } \\
\text { Voudouri, MD, } \\
\text { PhD }\end{array}$ & $\begin{array}{l}\text { Ophthalmologic Center } \\
\text { "Voudouri Adamantia," } \\
\text { Athens, Greece }\end{array}$ & $\begin{array}{l}\text { Data collection, critical } \\
\text { comments during } \\
\text { manuscript revision }\end{array}$ \\
\hline $\begin{array}{l}\text { Christina } \\
\text { Zompola, MD }\end{array}$ & $\begin{array}{l}\text { "Attikon" University } \\
\text { Hospital Athens, Greece }\end{array}$ & $\begin{array}{l}\text { Data collection, critical } \\
\text { comments during } \\
\text { manuscript revision }\end{array}$ \\
\hline $\begin{array}{l}\text { Georgios } \\
\text { Magoufis, MD }\end{array}$ & $\begin{array}{l}\text { Metropolitan Hospital, } \\
\text { Piraeus, Greece }\end{array}$ & $\begin{array}{l}\text { Data collection, } \\
\text { critical comments } \\
\text { during manuscript } \\
\text { revision }\end{array}$ \\
\hline $\begin{array}{l}\text { Chrysa Arvaniti, } \\
\text { MD, PhD }\end{array}$ & $\begin{array}{l}\text { "Attikon" University } \\
\text { Hospital Athens, Greece }\end{array}$ & $\begin{array}{l}\text { Critical comments } \\
\text { during manuscript } \\
\text { revision }\end{array}$ \\
\hline $\begin{array}{l}\text { Anastasios } \\
\text { Bonakis, MD, } \\
\text { PhD }\end{array}$ & $\begin{array}{l}\text { "Attikon" University } \\
\text { Hospital Athens, Greece }\end{array}$ & $\begin{array}{l}\text { Critical comments } \\
\text { during manuscript } \\
\text { revision }\end{array}$ \\
\hline $\begin{array}{l}\text { Georgios } \\
\text { Tsivgoulis, MD, } \\
\text { PhD, MSc, FESO }\end{array}$ & $\begin{array}{l}\text { "Attikon" University } \\
\text { Hospital Athens, Greece }\end{array}$ & $\begin{array}{l}\text { Data collection, } \\
\text { drafting the } \\
\text { manuscript }\end{array}$ \\
\hline \multicolumn{3}{|l|}{ References } \\
\hline \multicolumn{3}{|c|}{$\begin{array}{l}\text { 1. Kolodny E, Fellgiebel A, Hilz MJ, et al. Cerebrovascular involvement in Fabry disease: } \\
\text { current status of knowledge. Stroke 2015;46:302-313. }\end{array}$} \\
\hline \multicolumn{3}{|c|}{$\begin{array}{l}\text { 2. Brouns R, Thijs V, Eyskens F, et al. Belgian Fabry study: prevalence of Fabry disease in a } \\
\text { cohort of } 1000 \text { young patients with cerebrovascular disease. Stroke } 2010 ; 41: 863-868 \text {. }\end{array}$} \\
\hline
\end{tabular}




\section{Neurology}

\section{Teaching NeuroImages: An uncommon cause of carotid artery dissection: Fabry disease}

Aikaterini Theodorou, Lina Palaiodimou, Panagiotis Kokotis, et al. Neurology 2020;95;e2711-e2713 Published Online before print August 14, 2020

DOI 10.1212/WNL.0000000000010650

This information is current as of August 14, 2020

Updated Information \& Services

References

Subspecialty Collections

Permissions \& Licensing

Reprints including high resolution figures, can be found at: http://n.neurology.org/content/95/19/e2711.full

This article cites 2 articles, 2 of which you can access for free at: http://n.neurology.org/content/95/19/e2711.full\#ref-list-1

This article, along with others on similar topics, appears in the following collection(s):

All Cerebrovascular disease/Stroke

http://n.neurology.org/cgi/collection/all_cerebrovascular_disease_strok e

Carotid artery dissection

http://n.neurology.org/cgi/collection/carotid_artery_dissection CT

http://n.neurology.org/cgi/collection/ct

MRI

http://n.neurology.org/cgi/collection/mri

Stroke in young adults

http://n.neurology.org/cgi/collection/stroke_in_young_adults

Information about reproducing this article in parts (figures,tables) or in its entirety can be found online at:

http://www.neurology.org/about/about_the_journal\#permissions

Information about ordering reprints can be found online:

http://n.neurology.org/subscribers/advertise

Neurology ${ }^{\circledR}$ is the official journal of the American Academy of Neurology. Published continuously since 1951, it is now a weekly with 48 issues per year. Copyright @ 2020 American Academy of Neurology. All rights reserved. Print ISSN: 0028-3878. Online ISSN: 1526-632X.

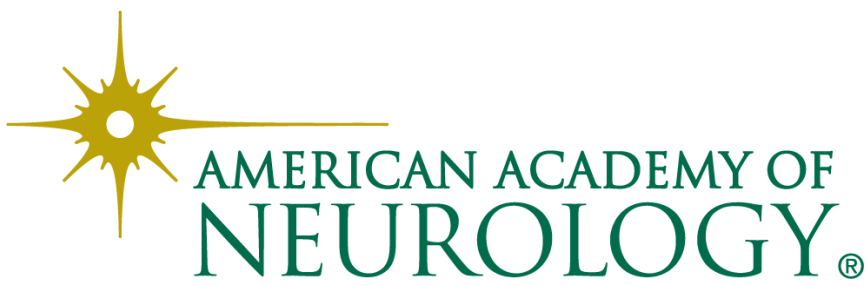

